\title{
Freisetzung kinetischer Energie und Hammond-Postulat bei der intramolekularen aromatischen Substitution in 2-Stilbazol-Ionen ${ }^{1)}$
}

\section{Ralf Schubert ${ }^{\mathrm{a}}$, Devalla Venkata Ramana ${ }^{\mathrm{b}}$ und Hans-Friedrich Grützmacher ${ }^{\mathrm{a} *}$}

Fakultät für Chemie, Universität Bielefeld ${ }^{a}$, Universitätsstraße, D-4800 Bielefeld 1, und Department of Chemistry, Indian Institute of Technology ${ }^{\mathrm{b}}$, Madras 600036, Indien

Eingegangen am 20. März 1980

In den Massenspektren der 2-Stilbazole $1 \mathrm{a}-1 \mathrm{~g}$, sowie des 3- und 4-Stilbazols ( 2 bzw. 3) werden durch Abspaltung eines $\mathrm{H}$-Atoms oder des Substituenten mit hoher Intensität $\mathrm{C}_{13} \mathrm{H}_{10} \mathrm{~N}^{+}{ }^{+}$-Ionen, $m / z=180$, gebildet. Für metastabile Molekül-Ionen von $1 \mathrm{~g}-1 \mathrm{~g}, 2$ und 3 ist dies die einzige Zerfallsreaktion. Durch einen Vergleich der MI- und CA-Spektren der $\mathrm{C}_{13} \mathrm{H}_{10} \mathrm{~N}^{{ }^{+}}$-Ionen ergibt sich, daß bei den 2-Stilbazolen 1a-1g 4a-Azoniaphenanthren-Ionen durch eine intramolekulare Substitution in den Molekül-Ionen entstehen. Die Abhängigkeit des Energiebedarfs und der Energie-Umsetzung bei dieser Reaktion von der Abgangsgruppe wird durch Messungen der Ionisations- und Auftrittsenergien sowie der bei der Reaktion freigesetzten kinetischen Energie untersucht. Danach verläuft die Reaktion uber eine Zwischenstufe $\mathbf{Z}$ mit tetrakoordiniertem $\mathbf{C}$ Atom in der ortho-Position des Phenylringes, deren Bildung die Aktivierungsenergie der Gesamtreaktion bestimmt. $\mathbf{Z}$ entsteht als chemisch aktivierte Spezies und besitzt gegenüber einem Zerfall zu den Endprodukten überschüssige Energie. Die Aufteilung der überschüssigen Energie auf innere Energie und Translationsenergie der Reaktionsprodukte wird von der Abgangsgruppe bestimmt. Diese Abhängigkeit wird mit Hilfe eines quantitativen Hammond-Postulats durch die Position des Ubergangszustandes auf der Reaktionskoordinate beschrieben.

Kinetic Energy Release and Hammond Postulate During Intramolecular Aromatic Substitution in 2-Stilbazole Ions 1 )

In the mass spectra of 2-stilbazoles $1 \mathrm{a}-1 \mathrm{~g}$ and 3 - and 4-stilbazoles ( 2 resp. 3), $\mathrm{C}_{13} \mathrm{H}_{10} \mathrm{~N}^{7+}$ ions, $m / z=180$, are formed with high intensities by loss of a $\mathrm{H}$-atom or of a substituent. This is the only fragmentation reaction available to metastable molecular ions of $1 \mathrm{a}-1 \mathrm{~g}, 2$, and 3 . Comparison of $\mathrm{MI}$ and $\mathrm{CA}$ spectra of the $\mathrm{C}_{13} \mathrm{H}_{10} \mathrm{~N}^{1+}$ ions shows, that $4 \mathrm{a}$-azoniaphenanthrene ions a arise from the 2-stilbazoles $1 \mathrm{a}-\mathbf{1 g}$ by an intramolecular substitution reaction of the molecular ions. The dependence of the energy requirements of this reaction has been studied by measurements of ionization and appearance energies and of the release of kinetic energy during the reaction. From this it follows that the reaction occurs via an intermediate $\mathbf{Z}$ with a tetracoordinated $\mathbf{C}$-atom at the ortho-position of the phenyl group; the formation of $\mathbf{Z}$ determines the activation energy of the total reaction. $\mathbf{Z}$ is formed as a chemically activated species and contains excess energy with respect to fragmentation into the reaction products. The partitioning of the excess energy between internal and translational energy of the reaction products depends on the leaving group. This dependence is described with the aid of a quantitative Hammond postulate by the position of the transition state at the reaction coordinate.

Massenspektrometrische Techniken zur Untersuchung metastabiler Ionen ermöglichen es, unimolekulare Reaktionen komplexer organischer Ionen in der Gasphase genauer als bisher zu untersuchen und interessante Hinweise auf den Ablauf chemischer Elementarreaktionen zu 
gewinnen 2). Die Zerfallsprodukte metastabiler Ionen besitzen in vielen Fällen eine überschüssige kinetische Energie $T$, die aus der Breite der Signale für die betreffenden Zerfallsreaktionen in einem mass analyzed kinetic energy-Spektrum (MIKE-Spektrum) gut bestimmt werden kann ${ }^{3)}$.

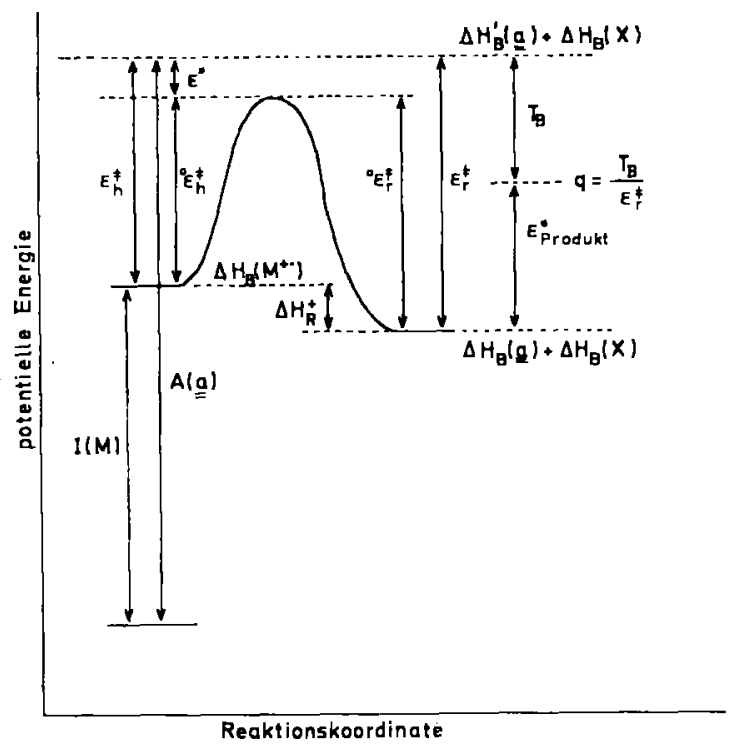

Abb. 1. Schematische Darstellung der potentiellen Energie während einer unimolekularen massen-

$I(\mathrm{M}) \quad=$ lonisierungsenergie; spektrometrischen Fragmentierung

$A(\mathbf{a}) \quad=$ Auftrittsenergie des Fragment-Ions a;

$\Delta H_{\mathrm{B}}\left(\mathrm{M}^{+*}\right), \Delta H_{\mathrm{B}}(\mathrm{a}), \Delta H_{\mathrm{B}}(\mathrm{X})$

= Bildungsenthalpien des Molekül-lons, des Fragment-Ions a und des Radikals X;

$\Delta H_{\mathrm{B}}^{\prime}(\mathrm{a})=$ scheinbare Bildungsenthalpie von $\mathbf{a} ;$

${ }^{0} \varepsilon_{\mathrm{h}}^{ \pm},{ }^{0} \varepsilon_{\mathrm{r}}^{\neq}=$thermochemische Aktivierungsenergien der Hin- und Rückreaktion;

$\varepsilon_{\mathrm{h}}^{*}, \varepsilon_{\mathrm{r}}^{*}=$ experimentelle Aktivierungsenergien der Hin- und Rückreaktion;

$\varepsilon^{*} \quad=$ frei fluktuierende Überschußenergie des aktivierten Komplexes;

$\varepsilon_{\text {Produkı }}^{*}=$ innere Anregungsenergie der Produkte;

$T_{\mathrm{B}} \quad=$ freigesetzte Translationsenergie der Produkte;

$q \quad=$ Energicaufteilungsquotient

Das in Abb. 1 schematisch wiedergegebene Energiediagramm der unimolekularen Reaktion eines Molekül-Ions läßt erkennen, daß die kinetische Energie $T$ der Reaktionsprodukte aus der Aktivierungsenergie $\varepsilon_{\mathrm{r}}^{\neq}$der Ruckreaktion stammt, die beim Fortschreiten der Hinreaktion vom aktivierten Komplex freigesetzt wird. Die experimentelle Größe $\varepsilon_{\mathrm{r}}^{*}$ setzt sich aus zwei Beiträgen zusammen: der thermochemischen Aktivierungsenergie $\varepsilon_{\mathrm{r}}^{\circ}$ der Rückreaktion, entsprechend der Differenz der potentiellen Energie von aktiviertem Komplex und Zerfallsprodukten in den Grundzuständen, und der frei im aktivierten Komplex fluktuierenden Anregungsenergie $\varepsilon^{*}$, die dem System eine für massenspektrometrische Messungen ausreichende Zerfallsgeschwindigkeit verleihe (,kinetische Verschiebung"). Bei den langsamen Reaktionen metastabiler Ionen ist $\varepsilon^{*}$ in der Regel klein, so daß für Prozesse mit großeren Werten für $\varepsilon_{\mathrm{r}}^{\circ}$ in guter Năherung $\varepsilon_{\mathrm{r}}^{*} \approx \varepsilon_{\mathrm{r}}^{\circ}$ gilt ${ }^{3,4}$ ). Welcher Anteil von $\varepsilon_{\mathrm{r}}^{*}$ (bzw. $\varepsilon_{\mathrm{r}}^{o}$ ) bei der Hinreaktion als kinetische Energie $T$ in den Zerfallspro- 
dukten auftritt, wird offensichtlich von fundamentalen Eigenschaften der Übergangszustände und den Bewegungen der aktivierten Komplexe längs der Reaktionskoordinate bestimmt ${ }^{5}$. Für einen bestimmten Prozeß kann bei bekanntem $T$ und $\varepsilon_{\mathrm{r}}^{*}$ der Energieaufteilungsquotient $q=T / \varepsilon_{\mathrm{r}}^{*}$ experimentell ermittelt werden. In einer Serie analoger Reaktionen mit strukturellen Änderungen in den Edukt-Ionen sollten sich entsprechende Änderungen der Úbergangszustände in den Werten für $q$ widerspiegeln, so daß sich die Möglichkeit ergibt, Modellvorstellungen über den Ablauf chemischer Reaktionen auch am Beispiel komplizierter organischer Ionen zu prüfen.

In vorhergehenden Arbeiten war gezeigt worden, daß bei intramolekularen aromatischen Substitutionsreaktionen in den Molekül-Ionen von Benzalacetonen ${ }^{6)}$, 2-Benzoylpyridinen ${ }^{7)}$ und 1-Pheny]-1-(2-pyridyl)ethylenen ") ein deutlicher Einfluß der Abgangsgruppe bei der Verteilung von $\varepsilon_{\mathrm{r}}^{*}$ auf kinetische Energie $T$ und Anregungsenergie der Reaktionsprodukte besteht, der entsprechend dem Hammond-Postulat durch eine Verschiebung der Übergangszustände auf der Reaktionskoordinate erklärt werden kann. In dieser Arbeit wird über den Einfluß der Abgangsgruppen auf die intramolekulare Substitutionsreaktion in den trans-2-Stilbazolen $1 \mathbf{a}-1 \mathrm{~g}$ berichtet.

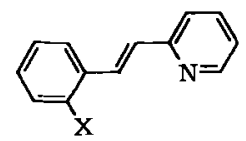

\begin{tabular}{|c|c|c|c|}
\hline & $x$ & & $x$ \\
\hline 1a & $\mathrm{H}$ & 1e & $\mathrm{Br}$ \\
\hline b & $F$ & I & I \\
\hline c & $\mathrm{CH}_{3}$ & g & $\mathrm{NO}_{2}$ \\
\hline d & $\mathrm{Cl}$ & & \\
\hline
\end{tabular}

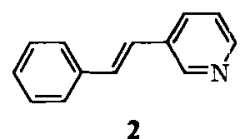

2

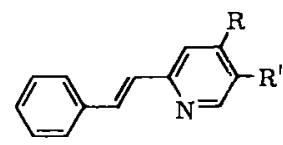

\begin{tabular}{l|ll} 
& $\mathrm{R}$ & $\mathrm{R}^{\prime}$ \\
\hline $\mathbf{4}$ & $\mathrm{H}$ & $\mathrm{Cl}$ \\
$\mathbf{5}$ & $\mathrm{Cl}$ & $\mathrm{H}$
\end{tabular}

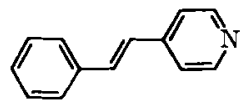

3

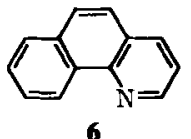

6

\section{Massenspektren}

Die 70-eV-Massenspektren des trans-2-Stilbazols (1a), trans-3-Stilbazols (2) und trans-4-Stilbazols (3) enthaiten nur wenige intensive Peaks. Neben den Molekül-Ionen werden bei allen drei Verbindungen in größerer Menge nur die Fragment-Ionen $\mathrm{M}-\mathrm{H}]^{+}, m / z=180$, und $\mathrm{M}-\mathrm{H}-\mathrm{HCN}^{+}, m / z=152$, gebildet, wobei sich in Abhängigkeit von der Stellung des $\mathbf{N}$-Atoms deutliche Intensitätsverschiebungen ergeben (Tab. 1). Die bei $1 \mathrm{a}$ überragende Intensität der $\mathbf{M}-\mathbf{H}\rceil^{+}$-Ionen läßt sich durch die leichte Bildung von 4a-Azoniaphenanthren-Ionen (a) $\mathrm{C}_{13} \mathrm{H}_{10} \mathrm{~N}{ }^{+}$(Schema 1, Weg a) erklären, während aus den Molekül-Ionen von 2 und 3 durch $\mathrm{C}-\mathrm{C}$-Verknüpfung und Wasserstoff-Umlagerung die isomeren protonierten Azaphenanthrene b' und b" entstehen (Schema 1). Derartige an die Photocyclisierung erinnernde $\mathrm{C}-\mathrm{C}$-Verknüpfungen sind aus den Massenspektren von Stilbenen bekannt ${ }^{8)}$. Im Fall der Molekül-Ionen von 1 a führen sie zu dem $\mathbf{M}-\mathrm{H}^{\dagger+}$-Ion b (Schema 1, Weg b).

Nur bei 1 a können die $\mathrm{C}_{13} \mathrm{H}_{10} \mathrm{~N} l^{+}$-Ionen a als Folge einer einfachen Substitutionsreaktion an der Phenylgruppe durch das $\mathrm{N}$-Atom des Phenylringes entstehen, und die erhohte Intensität der Ionen $\mathrm{C}_{13} \mathrm{H}_{10} \mathrm{~N}^{+}$zeigt, daß of fenbar ein energetisch besonders günstiger Prozeß vorliegt. Dies folgt auch aus den unterschiedlichen Intensitätsverhältnissen $M-H^{l+} / M-D^{l^{+}}$in den Massenspektren der im Phenylring pentadeuterierten Derivate von $1 \mathrm{a}$ und 3. Das pentadeuterierte 3 
liefert bei $70 \mathrm{eV}$ einen Wert von 0.83, der bei Absenken der Energie der Stoßelektronen auf $15 \mathrm{eV}$ leicht ansteigt. Dies kann durch Wanderungen der $\mathrm{H}$ - und D-Atome vor dem Zerfall zu den Ionen $b^{\prime \prime}$ erklärt werden. Das für pentadeuteriertes $1 \mathrm{a}$ bei $70 \mathrm{eV}$ gefundene Verhältnis $\mathrm{M}-\mathrm{H}^{+} / \mathrm{M}-\mathrm{Dl}^{+}=0.28$ ist demgegenüber zugunsten einer $\mathrm{D}$-Abspaltung verschoben. Bei einer Elektronenenergie von $15 \mathrm{eV}$ beträgt es nur noch 0.01 , so daß unter den Fragmentierungen der wenig angeregten Molekül-Ionen eine für die Substitutionsreaktionen erwartete D-Abspaltung stark überwiegt.

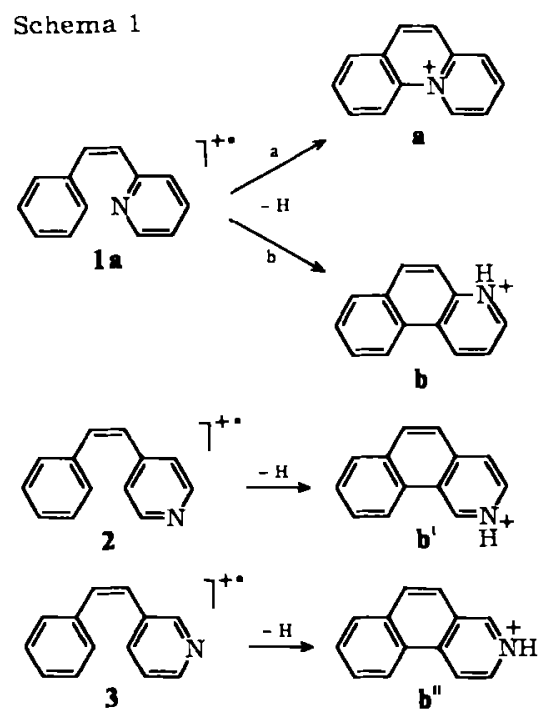

Tab. 1. 70-eV-Massenspektren a) der Stilbazole. Relative Intensitäten (\%)

\begin{tabular}{lrrrrrrrrrrrr}
\hline$m / z$ & $\mathbf{1}$ a & $\mathbf{1 b}$ & $\mathbf{1 c}$ & $\mathbf{1 d}$ & $\mathbf{1 e}$ & $\mathbf{1 f}$ & $\mathbf{1 g}$ & $\mathbf{2}$ & $\mathbf{3}$ & $\mathbf{4}$ & $\mathbf{5}$ \\
\hline $\mathbf{M}$ & & 14 & 28 & 11 & $16^{\text {b) }}$ & $14^{\text {b) }}$ & $\mathbf{8}$ & 5 & 52 & 64 & 14 b) & 10 b) \\
$\mathbf{M}-1$ & $(-)$ & $\mathbf{6 6}$ & 23 & $10^{\text {b }}$ & 7 b) & 2 & 2 & $(-)$ & $(-)$ & $100^{\text {b) }}$ & $100^{\text {b) }}$ \\
180 & 100 & 100 & 100 & 100 & 100 & 100 & 100 & 100 & 100 & 5 & 3 \\
179 & 3 & 6 & 4 & 6 & 9 & 11 & 8 & 4 & 4 & 7 & 8 \\
178 & 5 & 8 & 5 & 9 & 11 & 14 & 12 & 5 & 7 & 8 & 8 \\
153 & 2 & 1 & 2 & 2 & 2 & 3 & 4 & 3 & 8 & 3 & 2 \\
152 & 10 & 4 & 9 & 10 & 13 & 20 & 15 & 5 & 13 & 5 & 5 \\
151 & 3 & 5 & 3 & 5 & 5 & 8 & 7 & 22 & 37 & 2 & 4 \\
128 & 3 & 1 & 2 & 2 & 2 & 3 & 4 & 2 & 4 & 1 & 1 \\
127 & 2 & 1 & 2 & 2 & 2 & 3 & 7 & 4 & 7 & 1 & 1 \\
126 & 1 & 1 & 1 & 1 & 2 & 3 & 3 & 2 & 4 & 1 & 2 \\
79 & 3 & 8 & 9 & 4 & 4 & 3 & 21 & 1 & 1 & 5 & 5 \\
78 & 4 & 9 & 6 & 6 & 6 & 7 & 34 & 4 & 4 & 5 & 6 \\
77 & 5 & 2 & 5 & 4 & 6 & 15 & 15 & 6 & 10 & 3 & 4 \\
76 & 2 & 2 & 2 & 3 & 3 & 9 & 7 & 6 & 10 & 5 & 5 \\
75 & 1 & 3 & 2 & 3 & 3 & 7 & 3 & 2 & 4 & 4 & 5 \\
74 & 1 & 1 & 1 & 1 & 1 & 4 & 1 & - & 2 & 1 & 2 \\
\hline
\end{tabular}

a) ${ }^{13} \mathrm{C}$-korrigiert. - b) Summe der Isotopenpeaks. 
Im Einklang mit der bevorzugten Bildung der Ionen a aus den Molekül-Ionen von 1a sind die Massenspektren der im Phenylring substituierten 2-Stilbazole. Nur bei orthoStellung des Substituenten in $\mathbf{1 b}-\mathbf{1 g}$ werden die Massenspektren vom Peak der Ionen $\mathbf{M}-\mathbf{X}^{\dagger+}, m / z=180$, beherrscht, während zugleich die Intensitäten der $\mathbf{M}-\mathrm{H}^{{ }^{+}}$Ionen absinken (Tab. 1). Bei meta- oder para-Stellung der Substituenten, wie bei $m$ und $p$-Chlor-2-stilbazol ( 4 bzw. 5, s. Tab. 1), werden die Basis-Peaks der Massenspektren dagegen durch die Ionen $\mathrm{M}-\mathrm{H}^{{ }^{+}}$geliefert; die Abspaltung der Substituenten aus den Molekül-Ionen ist nur von untergeordneter Bedeutung. Daß aus 1a-1g die gleichen $\mathrm{C}_{13} \mathrm{H}_{10} \mathrm{~N}^{+}$-Ionen $\mathrm{a}, m / z=180$, entstehen, läßt sich durch einen Vergleich der Metastabilen-Ionen-Spektren (MI-Spektren ${ }^{9)}$ ) beweisen, die mit Hilfe der Linked-Scan B/E-Technik ${ }^{10)}$ und der DADI-Technik ${ }^{11)}$ erhalten werden. Metastabile $\mathrm{C}_{13} \mathrm{H}_{10} \mathrm{~N}^{1+}$ Ionen aus allen Stilbazolen fragmentieren sowohl im 1. als auch im 2. feldfreien Raum eines VG-ZAB-2F-Massenspektrometers zu Produkt-Ionen $m / z=179,178,154,153$ und 152. Die Signale für die Bildung der Ionen $\mathrm{m} / z=179$ und 178 werden durch andere Zerfallsreaktionen im Massenspektrometer gestört ${ }^{12)}$ und eignen sich daher nicht für einen Vergleich der MI-Spektren. Für die Peakgruppe $m / z=154-152$ werden für metastabile Ionen a aus 1a-1g bei Messung im 1. und 2. feldfreien Raum innerhalb der

Tab. 2. MI-Spektren der $\mathrm{C}_{13} \mathrm{H}_{10} \mathrm{~N}^{7}$ - Ionen, $m / z=180$

a) durch Zerfalle im 1. feldfreien Raum, b) durch Zerfälle im 2. feldfreien Raum

\begin{tabular}{crrrrrrrrr}
\hline $\begin{array}{c}\text { a) } \\
m / z\end{array}$ & $\mathbf{1 a}$ & $\mathbf{1 b}$ & $\mathbf{1 d}$ & $\mathbf{1 e}$ & 1f & $\mathbf{1 g}$ & $\mathbf{2}$ & $\mathbf{3}$ & $\mathbf{6}$ \\
\hline 179 & 415 & 775 & 610 & 560 & 615 & 925 & 515 & 200 & - \\
178 & 102 & 111 & 122 & 100 & 105 & 110 & 136 & 55 & - \\
154 & 6 & 9 & 5 & 6 & 6 & 14 & 9 & 13 & 20 \\
153 & 30 & 29 & 27 & 27 & 26 & 29 & 100 & 100 & 100 \\
152 & 100 & 100 & 100 & 100 & 100 & 100 & 57 & 50 & 60 \\
151 & 15 & 16 & 14 & 15 & 15 & 14 & 8 & 7 & 13 \\
\hline \multirow{2}{*}{ b) } & $1 \mathbf{a}$ & $\mathbf{1 b}$ & $1 \mathbf{1 d}$ & $\mathbf{1 e}$ & $\mathbf{1 f}$ & $\mathbf{1 g}$ & $\mathbf{2}$ & $\mathbf{3}$ & $\mathbf{6}$ \\
$m / z$ & & & & & & & & & \\
\hline 179 & 1240 & 850 & 810 & 700 & 700 & 840 & 430 & 410 & - \\
178 & 120 & 170 & 145 & 145 & 140 & 140 & 80 & 45 & - \\
153 & 100 & 100 & 100 & 100 & 100 & 100 & 100 & 100 & 100 \\
152 & 83 & 97 & 95 & 104 & 98 & 88 & 27 & 24 & 50 \\
151 & 10 & 17 & 13 & 15 & 14 & 14 & 7 & 5 & 15 \\
\hline
\end{tabular}

Fehlergrenzen identische Intensitätsmuster erhalten, die sich deutlich von denen metastabiler $\mathrm{C}_{13} \mathrm{H}_{10} \mathrm{~N}^{1+}$--Ionen aus 2 und 3 unterscheiden (Tab. 2). Eine ähnliche Intensitätsverteilung wie bei 2 und 3 mit der Bildung der Ionen $m / z=153$ als Hauptprodukt wird auch erhalten, wenn $\mathrm{C}_{13} \mathrm{H}_{10} \mathrm{~N}^{\top}$ - -Ionen durch Protonierung von 4-Azaphenanthren (6) in der Gasphase des Massenspektrometers mittels chemischer Ionisation erzeugt werden. Danach erscheint die Bildung der Produkt-Ionen $\mathrm{m} / \mathrm{z}=153$ charakteristisch für den Zerfall metastabiler $\mathrm{C}_{13} \mathrm{H}_{10} \mathrm{~N}^{\top}$ - - Ionen mit der Struktur von protonierten Azaphenanthrenen (b-b") zu sein, während die 4a-Azoniaphenanthren-Ionen a stärker zu Produkt-Ionen $m / z=152$ zerfallen ${ }^{13)}$. 
Tab. 3. CA-Spektren der $\mathrm{C}_{13} \mathrm{H}_{10} \mathrm{~N}^{\top *}$-Ionen, $m / z=180$, durch Stoßaktivierung im 1. feldfreien Rauma)

\begin{tabular}{rrrrrrrrrr}
\hline$m / z$ & $\mathbf{1 a}$ & $\mathbf{1 b}$ & $\mathbf{1 d}$ & $\mathbf{1} \mathbf{e}$ & $\mathbf{1 f}$ & $\mathbf{1 g}$ & $\mathbf{2}$ & $\mathbf{3}$ & $\mathbf{6}$ \\
\hline 179 & $(40)$ & $(270)$ & $(180)$ & $(110)$ & $(90)$ & $(550)$ & $(30)$ & $(110)$ & $(90)$ \\
178 & $(60)$ & $(210)$ & $(220)$ & $(120)$ & $(110)$ & $(350)$ & $(\mathbf{4 0})$ & $(90)$ & $(\mathbf{4 3 )})$ \\
177 & 15 & 24 & 18 & 14 & 11 & 24 & 7 & 17 & 12 \\
176 & $\mathbf{3}$ & 6 & 6 & 2 & 3 & 6 & 6 & 4 & 5 \\
166 & 2 & $\mathbf{3}$ & $\mathbf{3}$ & 2 & 2 & 3 & 2 & 2 & 4 \\
165 & 4 & 6 & 5 & 4 & 3 & 6 & 1 & 1 & 3 \\
164 & 3 & 4 & 3 & 3 & 3 & 4 & 3 & 3 & 4 \\
154 & $(3)$ & $(4)$ & $(4)$ & $(3)$ & $(3)$ & $(14)$ & $(4)$ & $(6)$ & $(<1)$ \\
153 & $(15)$ & $(26)$ & $(22)$ & $(20)$ & $(18)$ & $(43)$ & $(21)$ & $(29)$ & $(7)$ \\
152 & $(51)$ & $(62)$ & $(88)$ & $(81)$ & $(75)$ & $(139)$ & $(79)$ & $(108)$ & $(37)$ \\
151 & $(23)$ & $(22)$ & $(30)$ & $(28)$ & $(28)$ & $(40)$ & $(35)$ & $(45)$ & $(43)$ \\
150 & 9 & $\mathbf{8}$ & 12 & 12 & 12 & 12 & 14 & 18 & 17 \\
141 & 2 & 3 & 2 & 2 & 2 & 3 & 1 & 1 & $<1$ \\
140 & 6 & 6 & 4 & 4 & 4 & 5 & 6 & 4 & 4 \\
139 & 7 & 6 & 4 & 4 & 4 & 5 & 13 & 15 & 10 \\
138 & 1 & 2 & 1 & 1 & 1 & 1 & 2 & 3 & 1 \\
129 & 2 & 2 & 2 & 2 & 2 & 3 & 1 & 1 & $<1$ \\
128 & 15 & 14 & 20 & 20 & 20 & 21 & 7 & 7 & 3 \\
127 & 12 & 9 & 15 & 15 & 15 & 16 & 18 & 20 & 9 \\
126 & 10 & 8 & 10 & 11 & 11 & 11 & 14 & 16 & 13 \\
125 & 4 & 4 & 5 & 5 & 5 & 5 & 4 & 4 & 4 \\
115 & 1 & 1 & 1 & 1 & 1 & 1 & 2 & 2 & 2 \\
114 & 2 & 2 & 1 & 1 & 1 & 1 & 2 & 2 & 3 \\
113 & 2 & 2 & 2 & 2 & 2 & 1 & 3 & 2 & 4 \\
103 & 1 & 1 & 1 & 1 & 1 & 1 & 2 & 1 & 1 \\
102 & 4 & 3 & 4 & 4 & 4 & 3 & 5 & 3 & 3 \\
101 & 9 & 7 & $\mathbf{8}$ & 9 & 9 & 7 & 5 & 3 & 3 \\
100 & 2 & 2 & 2 & 2 & 2 & 1 & 1 & 1 & 1 \\
99 & 2 & 2 & 2 & 2 & 2 & 1 & 2 & 2 & 2 \\
98 & 2 & 1 & 1 & 2 & 2 & 1 & 1 & 1 & 1 \\
\hline
\end{tabular}

a) Werte in Klammern wegen Beiträgen aus unimolekularen Zerfällen nicht zur Normierung verwendet.

Ähnliche Unterschiede ergeben sich, wenn stabile $\mathrm{C}_{13} \mathrm{H}_{10} \mathrm{~N}^{1+}$-Ionen aus den verschiedenen Stilbazolen durch Stoßaktivierung während des Fluges durch das Massenspektrometer zum Zerfall angeregt werden (CA-Massenspektren ${ }^{14)}$ ). Dabei wird eine Vielzahl von Zerfallsprodukten beobachtet, die in den MIKE-CA-Spektren durch Überlappung der entsprechenden Signale zu breiten, mehr oder weniger strukturierten Peaks führen. Mit der linked-Scan-B/E-Technik werden jedoch für die Stoßaktivierung im 1. feldfreien Raum gut aufgelöste CA-Spektren erhalten (Tab. 3). Sie zeigen, daß auch die stabilen $\mathrm{C}_{13} \mathrm{H}_{10} \mathrm{~N}^{1+}$-Ionen a aus $1 \mathrm{a}-1 \mathrm{~g}$ identisch sind. Die stabilen $\mathrm{C}_{13} \mathrm{H}_{10} \mathrm{~N}^{{ }^{+}}$-Ionen aus 2 und 3 liefern dagegen in den CA-Spektren neben kleineren Unterschieden deutlich mehr Produkt-Ionen $m / z=139$ und weniger Produkt-lonen $m / z$ $=128$. Dies gilt auch für die aus 4-Azaphenanthren durch chemische Ionisation erzeugten $\mathrm{C}_{13} \mathrm{H}_{10} \mathrm{~N}^{1+}$-Ionen ${ }^{15}$. Diese Untersuchungen zeigen daher klar, daß in den verschiedenen Bereichen der Anregungsenergien aus den Molekül-Ionen von 1a-1g die 
gleichen Ionen $\mathrm{C}_{13} \mathrm{H}_{10} \mathrm{~N}^{1+}$ gebildet werden, die sich in ihrer Struktur von den protonierten Azaphenanthrenen $\mathbf{b}-\mathbf{b}^{\prime \prime}$ unterscheiden und den Ionen a entsprechen. Die Bildung von a erfolgt dabei durch die in Schema 1, Weg a, formulierte intramolekulare aromatische Substitutionsreaktion.

\section{Reaktionsenergien}

Für die Beschreibung der unimolekularen Zerfallsreaktion von Molekül-Ionen sind die Aktivierungsenergie der Hinreaktion $\varepsilon_{\mathrm{h}}^{*}$, die Aktivierungsenergie der Rückreaktion $\varepsilon_{\mathrm{I}}^{*}$ und die Reaktionsenthalpie $\Delta H_{\mathrm{R}}^{+}$die wichtigsten energetischen Größen (s. Abb. 1). Bei der Reaktion metastabiler Molekül-Ionen läßt sich $\varepsilon_{h}^{*}$ in guter Näherung durch die Differenz der Auftrittsenergie $A\left(\mathrm{~F}^{+}\right)$der Produkt-Ionen und der Ionisierungsenergie $I(\mathrm{M})$ der Ausgangsmoleküle wiedergegeben ${ }^{16)}$.

Tab. 4. Ionisierungsenergien $I(\mathrm{M})$ von Stilbazolen sowie Auftrittsenergien $A$ (a) und scheinbare Bildungsenthalpien der Ionen $\mathrm{m} / \mathrm{z}=180$

\begin{tabular}{|c|c|c|c|c|c|c|c|c|c|}
\hline & $1 \mathrm{a}$ & $1 \mathrm{~b}$ & $1 \mathrm{c}$ & $1 \mathrm{~d}$ & $1 \mathrm{e}$ & $1 \mathrm{f}$ & $1 \mathrm{~g}$ & 2 & 3 \\
\hline$I(\mathrm{M})^{\text {a) }}$ & 8.15 & 8.1 & 8.0 & 8.05 & 8.05 & 7.95 & 8.3 & 8.35 & 8.36 \\
\hline$A(\mathrm{a})^{\text {a) }}$ & 9.0 & 9.2 & 9.1 & 9.2 & 9.2 & 8.9 & 8.8 & 10.2 & 10.3 \\
\hline$\varepsilon_{h}^{*}$ b) & 0.8 & 0.9 & 1.1 & 1.1 & 1.1 & 1.0 & 0.5 & 1.8 & 1.7 \\
\hline$\Delta H_{\mathrm{B}}^{\prime}(\mathrm{a})^{\mathrm{b})}$ & 947 & 909 & 1002 & 1036 & 1106 & 1136 & 1094 & - & - \\
\hline$\Delta H_{\mathrm{B}}^{\prime}(\mathrm{a})_{\mathrm{c}}^{\mathrm{b}} \mathrm{b}_{\mathrm{r} r}$ & 888 & 892 & 935 & 1007 & 1073 & 1090 & 1027 & - & - \\
\hline
\end{tabular}

a) In eV. - b) In $\mathrm{kJ} \cdot \mathrm{mol}^{-1}$.

Diese Werte sind für die Bildung der $\mathrm{C}_{13} \mathrm{H}_{10} \mathrm{~N}^{\top}+$-Ionen aus $1 \mathrm{a}-1 \mathrm{~g}, 2$ und 3 in Tab. 4 aufgeführt. $I(\mathbf{1} \mathfrak{a})-I(\mathbf{1 g})$ folgt den Substituentenkonstanten $\sigma_{I \mathrm{P}}^{+}$von Bentley und Johnstone $^{17)}$, an der geringen Differenz von $I(\mathbf{l g})$ und $I(\mathbf{1 f})$ von nur $0.35 \mathrm{eV}$ wird jedoch der vergleichsweise geringe Substituenteneffekt auf das ausgedehnte $\pi$-Elektronensystem der 2-Stilbazole deutlich. Die Werte für $A$ (a) ändern sich in der Reihe 1a-1f trotz der erheblichen Unterschiede in den Dissoziationsenergien der während der Reaktion gespaltenen Bindung nicht deutlich, nur für $1 \mathrm{~g}$ mit $\mathrm{NO}_{2}$ als Abgangsgruppe wird ein kleinerer Wert erhalten. In Übereinstimmung mit den Schlußfolgerungen aus dem vorhergehenden Abschnitt zeigt sich, daß die Abspaltung eines H-Atoms aus den MolekülIonen von 1a energetisch deutlich günstiger ist als der Verlust eines H-Atoms aus den Molekül-Ionen von 2 und 3. Die Bildung der Ionen a erfordert mit Ausnahme von 1g durchweg eine Aktivierungsenergie von $1.0 \pm 0.1 \mathrm{eV}$, während für die $\mathrm{H}$-Abspaltung aus 2 und $31.7-1.8 \mathrm{eV}$ aufgewendet werden müssen.

Aus den Auftrittsenergien $A$ (a) für 1a-1g lassen sich scheinbare Bildungsenthalpien $\Delta H_{\mathrm{B}}^{\prime}(\mathbf{a})$ des $4 \mathrm{a}$-Azoniaphenanthren-Ions nach Gleichung (1) berechnen (Tab. 4) ${ }^{18)}$.

$$
\begin{aligned}
\Delta H_{\mathrm{B}}^{\prime}(\mathrm{a})=\Delta H_{\mathrm{B}}(\mathrm{a})+\varepsilon_{\mathrm{r}}^{*}=A(\mathrm{a})+ & \Delta H_{\mathrm{B}}\left(\mathrm{X}_{\mathrm{i}}\right)-\Delta H_{\mathrm{B}}(1 \mathrm{i}) \\
& (1 \mathrm{i} \doteq 1 \mathrm{a}-1 \mathrm{~g})
\end{aligned}
$$

$\Delta H_{\mathrm{B}}^{\prime}(\mathrm{a})$ weicht von der "wahren" Bildungsenthalpie $\Delta H_{\mathrm{B}}(\mathrm{a})$ durch den Beitrag der Aktivierungsenergie $\varepsilon_{\mathrm{r}}^{*}$ der jeweiligen Rückreaktion ab (s. Abb.1) und liefert daher nur obere Grenzwerte für $\Delta H_{\mathrm{B}}$ (a). Ein innerhalb der Reaktionsserie vermutlich unterschiedlicher Anteil von $\varepsilon_{\mathrm{r}}^{*}$ wird als kinetische Energie $T$ der Produkte freigesetzt und kann experimentell bestimmt werden. Durch 
Korrektur mit den bei den einzelnen Prozessen beobachteten maximalen Werten von $T$ werden die in der letzten Zeile der Tab. 4 aufgeführten $\Delta H_{\mathrm{B}}^{\prime}(\mathrm{a})_{\mathrm{corr}}$-Werte erhalten, die sich innerhalb der Serie dem wahren Wert für $\Delta H_{\mathrm{B}}$ (g) annahern sollten. Aus den kleinsten Werten ergibt sich daraus $\Delta H_{\mathrm{B}}(\mathrm{a})=890 \mathrm{~kJ} \cdot \mathrm{mol}^{-1}$, in befriedigender Übereinstimmung mit einem aus thermochemischen Daten abgeschätzten Wert von $895 \mathrm{~kJ} \cdot \mathrm{mol}^{-1}$ (s. exp. Teil).

In der Serie $1 \mathrm{a}-1 \mathrm{~g}$ werden weder für $\Delta H_{\mathrm{B}}^{\prime}(\mathbf{a})$ direkt noch nach Korrektur durch $T_{\mathrm{B}}$ konstante Werte erhalten. Dies zeigt klar, daß innerhalb der Serie $\varepsilon_{\mathrm{r}}^{*}$ nicht konstant ist. Zwischen $\Delta H_{B}^{\prime}(\mathbf{a})$ und den relativen Dissoziationsenergien $\Delta D(\stackrel{+}{\mathrm{C}}-\mathrm{X})$ der Bindungen zu den Substituenten in den Molekül-Ionen ${ }^{19}$ ergibt sich eine lineare Beziehung (Abb. 2). Dies ist zu erwarten, wenn in einer Reaktionsserie identische Produkt-Ionen mit konstanter Aktivierungsenergie $\varepsilon_{h}^{*}$ der Bildungsreaktionen entstehen ${ }^{20)}$. Bis auf $1 \mathbf{g}$, für das eine kleinere Aktivierungsenergie $\varepsilon_{\mathrm{h}}^{*}$ bestimmt wurde, ist dieser Fall hier offenbar gegeben. Mit Gleichung (1) folgt daraus auch eine lineare Beziehung zwischen $\Delta D(\mathrm{C}-\mathrm{X})$ und den Aktivierungsenergien $\varepsilon_{\mathrm{r}}^{*}$ der Rückreaktionen.

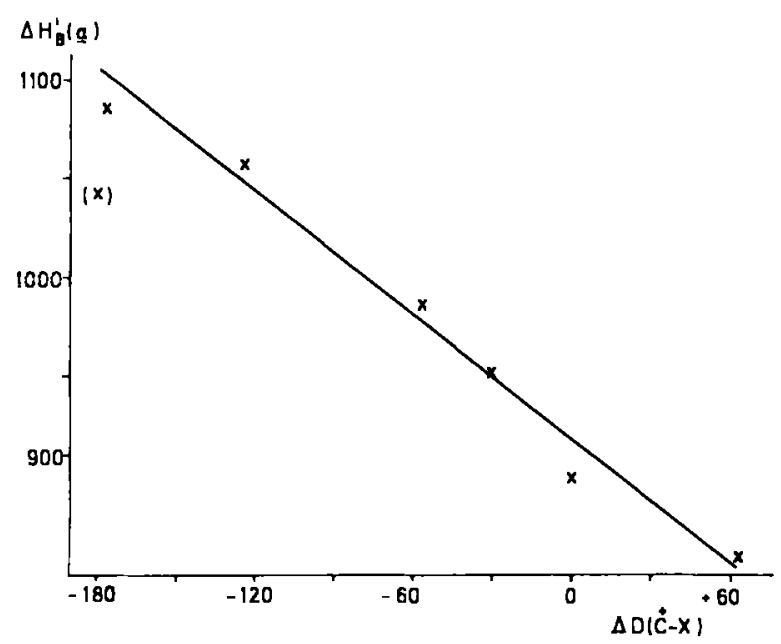

Abb. 2. Abhängigkeit der scheinbaren Bildungsenthalpie $\Delta H_{\mathrm{B}}^{\prime}(\mathrm{a})$ von der relativen Dissoziationsenergie $\Delta D(\stackrel{+}{\mathrm{C}}-\mathrm{X})$ der Substituenten in den Molekul-Ionen $1 \mathbf{a}-\mathbf{1} \mathbf{g}$

Mit $\Delta H_{\mathrm{B}}(\mathrm{g})=895 \mathrm{~kJ} \cdot \mathrm{mol}^{-1}$ können $\varepsilon_{\mathrm{\tau}}^{\neq}$(s. Gleichung (1)) und $\Delta H_{\mathrm{R}}^{+}$(s. Gleichung (2)) bestimmt werden (Tab. 5).

$$
\begin{aligned}
\Delta H_{\mathrm{R}}^{+} & =\Delta H_{\mathrm{B}}(\mathrm{a})+\Delta H_{\mathrm{B}}\left(\mathrm{X}_{\mathrm{i}}\right)-\Delta H_{\mathrm{B}}\left(1 \mathrm{i}^{+} \cdot\right) \\
& =\Delta H_{\mathrm{B}}(\mathrm{a})+\Delta H_{\mathrm{B}}\left(\mathrm{X}_{\mathrm{i}}\right)-\left(\Delta H_{\mathrm{B}}(\mathbf{1} \mathrm{i})+I(1 \mathrm{i})\right)
\end{aligned}
$$

Die intramolekulare Substitutionsreaktion in den Molekül-Ionen ist für das Fluorderivat 1 b und die Stammverbindung 1a deutlich endotherm, für das Methylderivat 1c mehr oder weniger thermoneutral und wird in der Reihe 1d-1g zunehmend exotherm. Entsprechend steigt die Aktivierungsenergie $\varepsilon_{\mathrm{r}}^{*}$ der Ruckreaktion von einem kleinen Wert bei 1 b zu großen Werten bei 1 e-1g. 
Tab. S. Reaktionsenthalpie $\Delta H_{\mathrm{R}}^{+}$, Aktivierungsenergie der Ruckreaktion $\varepsilon_{\mathrm{r}}^{*}$, freigesetzte kinetische Energie $T_{\mathrm{B}}$ und Energieaufteilungsquotient $q$ für Substituentenabspaltung aus 2-StilbazolIonen (Werte in $\mathrm{kJ} \cdot \mathrm{mol}^{-1}$ )

\begin{tabular}{|c|c|c|c|c|c|c|c|}
\hline & $1 \mathrm{a}$ & $1 \mathrm{~b}$ & $1 \mathrm{c}$ & $1 \mathrm{~d}$ & $1 \mathrm{e}$ & $1 \mathrm{f}$ & $1 \mathrm{~g}$ \\
\hline$\Delta H_{\mathrm{R}}^{+}$ & +30 & +85 & -2 & -32 & -100 & -151 & -151 \\
\hline$\varepsilon_{\mathrm{r}}^{F^{k}}$ & 52 & 14 & 107 & 141 & 211 & 241 & 199 \\
\hline$T_{\mathrm{B}}$ & 59 & 17 & 67 & 29 & 33 & 46 & 67 \\
\hline$q$ & 1.1 & 1.2 & 0.63 & 0.21 & 0.16 & 0.19 & 0.34 \\
\hline
\end{tabular}

Dieser systematische Verlauf für $\varepsilon_{\mathrm{r}}^{\neq}$findet sich für die freigesetzte kinetische Energie $T$ nicht wieder. Ermittelt man aus der Basisbreite der Signale für die Substituentenabspaltung in den MIKE-Spektren der Molekül-Ionen den maximalen Wert $T_{\mathrm{B}}{ }^{21)}$, so steigt dieser in der Reihe $1 \mathbf{b}-1 \mathbf{a}-1 \mathrm{c}$ mit $\varepsilon_{\mathrm{r}}^{*}$ zunächst an; bei weiter ansteigendem $\varepsilon_{\mathrm{r}}^{*}$ werden aber für $1 \mathrm{~d}$ und $1 \mathrm{e}$ kleinere und erst bei $1 \mathrm{f}$ und $1 \mathrm{~g}$ wieder großere Werte gefunden. Auch die Signalformen sind bei den einzelnen Reaktionen unterschiedlich. Für die F-Abspaltung aus den Molekül-Ionen von $1 \mathbf{b}$ wird ein Gauß-förmiger schmaler Peak erhalten, entsprechend einer statistischen Verteilung von $T^{22)}$, während für die Abspaltung von $\mathrm{H}$ bei $1 \mathrm{a}$ und $\mathrm{CH}_{3}$ bei $1 \mathrm{c}$ breite Signale mit flacher Kuppe entstehen. Die Substituentenabspaltungen aus den Molekül-Ionen von $1 \mathrm{~d}-1 \mathrm{~g}$ liefern dagegen breite, annähernd Gauß-förmige Signale. Die Übergangszustände der intramolekularen Substitutionsreaktionen der Ionen $1 \mathbf{a}-\mathbf{1 g}$ verhalten sich daher bei der Umwandlung überschüssiger Energie in Translationsenergie deutlich unterschiedlich. Dies läßt sich auch an den in Tab. 5 aufgeführten Energie-Aufteilungsquotienten $q=T_{\mathrm{B}} / \varepsilon_{\mathrm{r}}^{*}$ zeigen. Bei den Reaktionen von 1 a-1c mit $q>0.6$ wird $\varepsilon_{I}^{*}$ ganz oder zum größeren Anteil als Translationsenergie frei, während für $1 \mathrm{~d}-1 \mathrm{~g} q$ auf $0.2-0.3$ absinkt, und jetzt der gröBere Anteil von $\varepsilon_{\mathrm{r}}^{*}$ als Anregungsenergie in den Reaktionsprodukten verbleibt.

\section{Diskussion}

Eine uberraschende Eigenschaft der Substituentenabspaltung von den MolekülIonen substituierter 2-Stilbazole ist die innerhalb der Fehlergrenze konstante Aktivierungsenergie $\varepsilon_{\mathrm{h}}^{\neq}$für diesen Prozeß in der Reihe 1a-1f, obwohl die Dissoziationsenergien der Bindungen zur Abgangsgruppe zwischen $1 \mathrm{~b}$ und $1 \mathrm{f}$ um ca. $2.6 \mathrm{eV}$ differieren ${ }^{23)}$ und in den 70-eV-Massenspektren auch ein Intensitätsanstieg der Produkt-Ionen a beobachtet wird. Dieser Befund und der kleine Wert für $\varepsilon_{\mathrm{h}}^{*}$ von $1.0 \mathrm{eV}$ zeigen, daß die Substituenten aus den Molekül-Ionen nicht durch einen direkten Bindungsbruch abgespalten werden, sondern ein Nachbargruppeneffekt des Pyridinringes vorliegt. Dabei folgt aus dem unterschiedlichen Verhalten der isomeren Stilbazole 1a, 2 und 3 sowie der in verschiedenen Positionen der Phenylgruppe substituierten Derivate von 1a, daß bei der Bildung der Ionen a eine spezifische Wechselwirkung zwischen den N-Atomen und den ortho-Positionen der Phenylgruppe in $1 \mathrm{a}-1 \mathrm{~g}$ erfolgt, die als intramolekulare aromatische Substitutionsreaktion beschrieben werden kann. Die Substitution kann nicht einem Synchron-Prozeß mit direkter Verdrängung eines H-Atoms oder anderen Abgangsgruppen aus der ortho-Stellung der Phenylgruppe entsprechen, weil in diesem Fall ebenfalls ein merklicher Einfluß der Bindungsstärke zur Abgangsgruppe auf $\varepsilon_{\mathrm{h}}^{\neq}$be- 
obachtet werden sollte. Dem Schritt der Substituentenabspaltung muß daher mindestens ein weiterer Reaktionsschritt vorgelagert sein, der die Aktivierungsenergie der Gesamtreaktion bestimmt und nicht merklich von der Dissoziationsenergie der Substituenten-Bindung abhängt .

Für die Reaktion werden trans-2-Stilbazole eingesètzt; vor der Bildung von a muß daher eine trans $\rightarrow$ cis-Isomerisierung der Molekül-fonen erfolgen. Die Auftrittsenergie der Ionen a aus cis-Stilbazol ist mit $9.0 \pm 0.1 \mathrm{eV}$,jedoch die gleiche wie bei (trans-)-1a, so daß die trans $\rightarrow$ cis-Isomerisierung der 2-Stilbazol-Ionen als energiebestimmender Schritt ausscheidet. Es bleibt dann der Angriff des N-Atoms im Pyridinring auf die ortho-Position der Phenylgruppe in den 2-Stilbazol-Ionen unter Bildung der Zwischenstufe Z als Reaktionsschritt mit der höchsten Aktivierungsenergie (Schema 2). Die Bildung einer Zwischenstufe mit tetrakoordiniertem C-Atom am Reaktions-Zentrum entspricht dem Verlauf aromatischer Substitutionsreaktionen in kondensierten Phasen unter thermischen Gleichgewichtsbedingungen. Die Substituentenabspaltung aus den 2Stilbazol-Ionen bietet daher die Möglichkeit, eine verwandte Substitutionsreaktion in isolierten Spezies ohne Energieaustausch mit der Umgebung zu untersuchen.

Schema 2

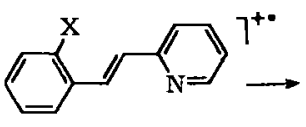

1a

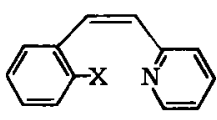

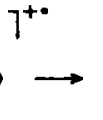

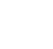

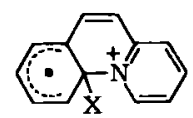

$\mathbf{Z}$

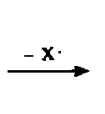

(2)

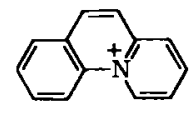

$\mathbf{n}$

Aus den Molekül-Ionen von 1a - $1 \mathrm{~g}$ entsteht $\mathbf{Z}$ durch chemische Aktivierung mit Anregungsenergien, die wegen des fehlenden Energieaustausches mit der Umgebung den Zerfall in die Reaktionsprodukte a und $X^{-}$bewirken. Entsprechend der RRKMTheorie ${ }^{24)}$ muß dabei die Zerfallsgeschwindigkeit mit der Energiedifferenz zwischen angeregtem $\mathbf{Z}$ und dem durch die Grundzustände von $\mathbf{a}$ und $\mathbf{X}$ - gegebenen Energieniveau zunehmen. Die zunächst überraschende Entkopplung der (konstanten) Aktivierungsenergie $\varepsilon_{\mathrm{h}}^{\neq}$für die Bildung von a und dessen Intensitäten in den Massenspektren von $1 \mathrm{a}-1 \mathrm{~g}$ ist nach diesem Mechanismus daher eine zwangsläufige Folge der Tatsache, daß massenspektrometrische Fragmentierungen unter den üblichen Bedingungen streng unimolekular sind.

Analoge Mechanismen wie für die Bildung von a aus 1a-1g sind bisher für die Abspaltung der Substituenten aus den Molekul-Ionen substituierter Benzalacetone ${ }^{6)}$, 1-Phenyl-1-(2-pyridyl)ethylene ${ }^{1)}$, 2-Benzoylpyridine ${ }^{7)}$ und $N, N$-Dimethyl- $N$ 'phenylformamidine ${ }^{25)}$ nachgewiesen worden. Für die Bildung von 2-Methylbenzopyrylium-lonen aus den Molekull-Ionen der substituierten Benzalacetone, die ebenso wie die intramolekulare Substitutionsreaktion in den 2-StilbazolIonen einen 6-gliedrigen Übergangszustand erfordert, wurden ebenfalls konstante, von der Dissoziationsenergie der Substituentenbindung unabhängige Werte für $\varepsilon_{h}^{*}$ gefunden, wobei das $o$ Nitroderivat ebenso wie 1g herausfällt ${ }^{6)}$. Dagegen ist $\varepsilon_{\mathrm{h}}^{*}$ für die Bildung cyclischer ProduktIonen durch eine Substitutionsreaktion mit 5 -gliedrigem Übergangszustand bei den MolekülIonen der 1-Phenyl-1-(2-pyridyl)ethylene ${ }^{1)}$ und der $N, N$-Dimethyl- $N^{\prime}$-phenylformamidine ${ }^{25)}$ schwach, bei den Molekül-Ionen der 2-Benzoylpyridine ") deutlich von der Dissoziationsenergie der Substituentenbindung abhängig. In allen Fällen bleibt $\varepsilon_{\mathrm{h}}^{*}$ jedoch klein $(<1.5 \mathrm{eV})$ und beträgt 
nur ein Bruchteil der Energien, die für die Abspaltung von Substituenten aus den Molekül-Ionen von Aromaten ohne einen Nachbargruppeneffekt eines Heteroatoms in der Seitenkette gemessen werden ${ }^{23)}$.

Die metastabilen Molekül-Ionen, von $1 \mathbf{a}-1 \mathrm{~g}$ besitzen eine für die Bildung von $\mathbf{Z}$ gerade ausreichende Anregungsenergie. Da die Bildung von $\mathbf{Z}$ jedoch den Energieaufwand für die Gesamtreaktion bestimmt, werden beim Zerfall von $\mathbf{Z}$ Überschußenergien frei, die unter den Bedingungen unimolekularer Reaktionen als Anregungsenergie $\varepsilon^{*}$ und/oder Translationsenergie $T$ in den Produkten a und $\mathrm{X}$ auftreten. Die Größe der Überschußenergie wird für den jeweiligen Prozeß durch die Aktivierungsenergie $\varepsilon_{r}^{*}$ der Rückreaktion bestimmt, die hier einer bimolekularen, unter Ringöffnung zum 2-Stilbazol-Ion verlaufenden Substitution des 4a-Azoniaphenanthren-Ions durch die Radikale $\mathbf{X}$ entspricht. In Bezug auf die Molekül-Ionen entsteht $\mathbf{Z}$ mit der gleichen Energie, so $\mathrm{da} ß \varepsilon_{\mathrm{r}}^{*}$ linear mit abnehmender Bindungsenergie des Substituenten ansteigen muß. Die in $\mathrm{Abb} .2$ wiedergegebene lineare Beziehung zwischen $\Delta H_{\mathrm{B}}^{\prime}(\mathrm{a})\left(=\Delta H_{\mathrm{B}}(\mathrm{a})+\varepsilon_{\mathrm{r}}^{\ddagger}\right)$ und $\Delta D(\stackrel{+}{\mathrm{C}}-\mathrm{X})$ ist daher ebenfalls eine Folge des in Schema 2 aufgeführten Mechanismus.

Der als kinetische Energie $T$ freigesetzte Anteil von $\varepsilon_{\mathrm{r}}^{*}$ kann bei massenspektrometrischen Reaktionen aus der Signalverbreiterung im MIKE-Spektrum des metastabilen Edukt-Ions bestimmt

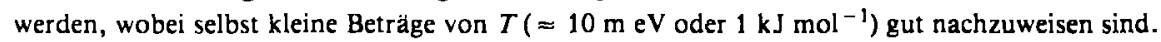
Eine statistische Verteilung von $\varepsilon_{\mathrm{r}}^{*}$ auf individuelle Werte von $\varepsilon^{*}$ und $T$ der Produkte, die schmale und Gaußförmige Signale in den MIKE-Spektren liefert, ist nur bei Reaktionen zu erwarten, für die $\varepsilon_{\mathrm{r}}^{*}$ überwiegend durch eine frei im aktivierten Komplex fluktuierende Überschußenergie (entsprechend dem „kinetic shift“ der Reaktion) bestimmt wird 2.3).

Ein schmales Gauß-förmiges Signal wird jedoch nur für die Abspaltung von $F$ aus metastabilen Molekül-Ionen von $\mathbf{1 b}$ beobachtet. Bei den übrigen 2-Stilbazolen zeigen abweichende Signalformen und erheblich größere Beträge von $T$ klar, daß bei diesen Prozessen $\varepsilon_{\mathrm{r}}^{\neq}$durch die in den chemischen Bindungen fixierte potentielle Energie des aktivierten Komplexes bestimmt wird. Die Umwandlung dieser Energie in Translationsenergie der Produkte wird beim Fortschreiten der Reaktion von der Reaktionsdynamik bestimmt ${ }^{5)}$. Den unterschiedlichen Werten für den Energieaufteilungs-Quotienten $q$ (Tab. 5) in der Reihe $1 \mathrm{a}-1 \mathrm{~g}$ entsprechen daher Unterschiede im dynamischen Verhalten der aktivierten Komplexe beim Zerfall in die Reaktionsprodukte. Dabei setzt sich die Gruppe 1a - 1c mit $q>0.6$ deutlich von der Gruppe $1 \mathrm{~d}-1 \mathrm{~g}$ mit $q<0.35 \mathrm{ab}$.

Diese Unterschiede bleiben auch unter Berücksichtigung der Fehler bei der Bestimmung von $q$ erhalten. Die Bildungsenthalpien der neutralen Verbindungen $1 \mathbf{a}-\mathbf{1 g}$ in der Gasphase wurden einheitlich aus Inkrementen ${ }^{18}$ ) berechnet, so daß im wesentlichen ein systematischer Fehler durch die Ungenauigkeit bei der Berechnung des Grundgerustes 1 a vorhanden ist. Der Fehler bei der Abschätzung von $\Delta H_{\mathrm{B}}(\mathrm{a})$ kann beträchtlich sein, da außer durch Vergleich mit $\Delta H_{\mathrm{B}}^{\prime}(\mathrm{a})_{\text {corr }}$ keine Kontrolle mit experimentellen Daten möglich ist. Dies ist aber ebenfalls ein systematischer Fehler, der die Einteilung von $1 \mathbf{a}-1 \mathrm{~g}$ in zwei Gruppen durch die $q$-Werte nicht verandert. Unter den ubrigen experimentellen Fehlern dominiert der Fehler von $\pm 0.1 \mathrm{eV}\left( \pm 10 \mathrm{~kJ} \mathrm{~mol}^{-1}\right)$ bei der Bestimmung von $A(\mathrm{a})$, der entsprechend für $\varepsilon_{\mathrm{r}}^{\neq}$gilt.

Es ist auffallend, daß die Einteilung in zwei Gruppen mit $q>0.6$ und Freisetzung eines hohen Anteils von $\varepsilon_{\mathrm{\tau}}^{*}$ als kinetische Energie sowie $q<0.35$ und Umwandlung des größeren Anteils von $\varepsilon_{\mathrm{r}}^{*}$ in innere Energie der Produkte mit der Einteilung in endotherme oder thermoneutrale Prozesse für $1 \mathrm{a}-1 \mathrm{c}$ einerseits und exotherme Prozesse fur $1 \mathrm{~d}-1 \mathrm{~g}$ andererseits zusammenfallt. Nach dem Hammond-Postulat ${ }^{26)}$ besitzen endotherme Reaktionen "späte" Übergangszustände, die in 
ihren Eigenschaften den Reaktionsprodukten ahnlich sind, während bei exothermen Reaktionen "frühe", den Edukten ähnliche Übergangszustände auftreten. Diese Beziehung kann nach Miller ${ }^{27)}$ quantitativ durch eine Funktion $X_{\mathbf{0}}^{*}$ ausgedrückt werden, die die Position des Úbergangszustandes einer Elementarreaktion auf der Reaktionskoordinate als Funktion der potentiellen Energie $U_{\mathrm{f}}$ der Reaktion (hier $\Delta H_{\mathrm{R}}^{+}$) und der Hobhe $U^{*}$ der Aktivierungsbarriere (hier $\varepsilon_{\mathrm{h}}^{*}$ ) wiedergibt (Gleichung (3)).

$$
X_{\mathrm{o}}^{*}=\frac{1}{2-\frac{U_{\mathrm{f}}}{U^{*}}}=\frac{1}{2-\frac{\Delta H_{\mathrm{R}}^{+}}{\varepsilon_{\mathrm{h}}^{*}}}
$$

Die intramolekulare Substitution in den Molekül-Ionen der 2-Stilbazole ist für eine Betrachtung mit Hilfe des Hammond-Postulats besonders geeignet, da sie außer bei $\mathbf{~ g ~}$ mit konstanter Aktivierungsenergie $\varepsilon_{\mathrm{h}}^{*}$ verlauft. Die Anwendung von Gleichung (3) liefert die in Tab. 6 angegebenen $X_{0}^{*}$-Werte; Abb. 3 zeigt ein damit konstruiertes Hammond-Diagramm der potentiellen Reaktionsenergie für 1a-1f.

Tab. 6. Position des Übergangszustandes $X_{0}^{*}$ und Energieaufteilungsquotient $q$ für Substituentenabspaltung aus 2-Stilbazol-Ionen

\begin{tabular}{cccccccc}
\hline & $1 \mathrm{~b}$ & $\mathbf{1 ~ a}$ & $\mathbf{1 c}$ & $\mathbf{1 d}$ & $1 \mathrm{e}$ & $\mathbf{1 f}$ & $\mathbf{1 g}$ \\
\hline$X_{\mathbf{0}}^{*}$ & 0.88 & 0.61 & 0.50 & 0.44 & 0.34 & 0.27 & 0.19 \\
$q^{*}$ & 1.2 & 1.1 & 0.63 & 0.21 & 0.16 & 0.19 & 0.34 \\
\hline
\end{tabular}

Man erkennt, wie sich die Position des Übergangszustandes mit zunehmender Exothermizităt von „spät" ( $\left.X_{0}^{*}=0.88\right)$ für 1 b zu „früh“ $\left(X_{0}^{*}=0.27\right)$ für 1 f verschiebt. Die Änderung in der Aufteilung von $\varepsilon_{\mathrm{r}}^{*}$ zugunsten der inneren Energie der Produkte erfolgt beim Úbergang von 1c mit $X_{\mathrm{o}}^{*}=0.50 \mathrm{zu} 1 \mathrm{~d} \mathrm{mit} X_{\mathrm{o}}^{*}=0.44$. Eine sprunghafte Änderung des Energieaufteilungsquotienten $q$ in Abhängigkeit von der Abgangsgruppe war auch bei den intramolekularen Substitutionsreaktionen in den Molekül-Ionen von Benzalacetonen $^{6}$, 1-Phenyl-1-(2-pyridyl)ethylenen ${ }^{1)}$ und 2-Benzoylpyridinen ${ }^{7)}$ beobachtet worden. Es ist bemerkenswert, daß in allen Fällen die Freisetzung eines großßeren Anteils von $\varepsilon_{\mathrm{r}}^{*}$ als Translationsenergie $T$ unterbleibt, wenn der Übergangszustand eine "fruhe“ Position mit $X_{0}^{*}<0.4$ besitzt. Man gewinnt daher den Eindruck, daß sich hier eine charakteristische Eigenschaft dieses Typs einer aromatischen Substitutionsreaktion zu erkennen gibt. Betrachtet man den Zerfall der Zwischenstufe Z, so entspricht einer Reaktion mit spätem Übergangszustand eine Energiehyperfläche, die erst spät zu den Reaktionsprodukten abfallt ("late down-hill surface" 28) mit einer entsprechend großen Dehnung der Substituentenbindung. Ein großer Anteil der Úberschußenergie von $\mathbf{Z}$ ist daher in dieser gedehnten Bindung lokalisiert und wird beim Zerfall als kinetische Energie frei. Bei einem frühen Übergangszustand und einer Energiehyperfläche mit entsprechend frühem Abfall zu den Reaktionsprodukten ("early down-hill surface “ 28) ist bis zur kritischen Konfiguration des aktivierten Komplexes nur eine kleine Aufweitung der Bindung zur Abgangsgruppe notwendig, so daß auch nur ein kleiner Anteil der Anregungsenergie in dieser gedehnten Bindung fixiert ist und als Translationsenergie frei wird. Entsprechende Beziehungen zwischen der Position der Aktivierungsbar- 
riere einer Reaktion und der Translationsenergie bzw. inneren Energie der reagierenden Teilchen sind aus experimentellen Untersuchungen zur Reaktionsdynamik einfacher Reaktionssysteme bekannt ${ }^{28)}$. Sie können nach den hier vorgelegten Ergebnissen direkt auf die Reaktionen großer organischer Ionen übertragen und durch massenspektrometrische Untersuchungen nachgewiesen werden.

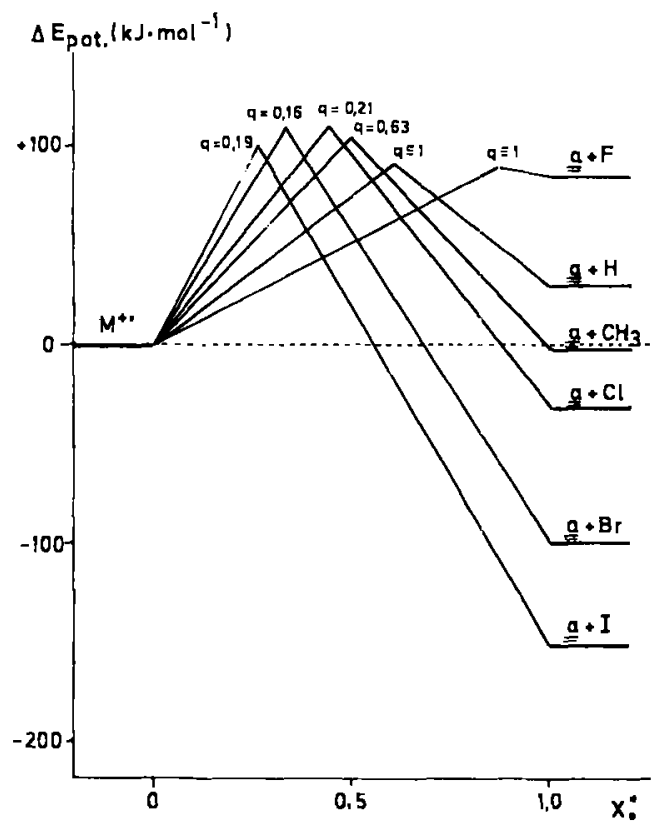

Abb. 3. Hammond-Diagramm der intramolekularen Substitution in den Molekül-Ionen $1 \mathrm{a}-1 \mathrm{~g}$ $\left(X_{0}^{*}=\right.$ Position des Übergangszustandes auf der Reaktionskoordinate; $q=$ Energieaufteilungsquotient)

Dem Fonds der Chemischen Industrie danken wir für die Unterstützung bei unseren Untersuchungen. Diese Arbeit ist ein Teil des Forschungsprojektes OZ 2141 der Universität Bielefeld. D. V. R. dankt dem Deutschen Akademischen Austausch-Dienst für die Finanzierung eines Gastaufenthaltes in Deutschland.

\section{Experimenteller Teil}

Schmelzpunkte (unkorrigiert): Leitz-Heizmikroskop 350. - IR: Perkin-Elmer Modell 377. Dünnschicht-Chromatographie: Kieselgelfolie GF 254 (Merck), Laufmittel Benzol/Essigester (10:1). - C,H,N-Analysen: Analytisches Labor der Fakultät für Chemie.

\section{Massenspektrometrische Messungen}

Die El-Massenspektren wurden mit einem Massenspektrometer Varian MAT 311A durch Direkt-Einlaß der Proben (Al-Tiegel, Probentemp. $20-60^{\circ} \mathrm{C}$ ) unter folgenden Bedingungen aufgenommen: Elektronenenergie $70 \mathrm{eV}$, Emissionsstrom $2 \mathrm{~mA}$, Beschleunigungsspannung $3 \mathrm{kV}$, Massenauflosungsvermogen $\approx 1000$, Temperatur der Ionenquelle $150^{\circ} \mathrm{C}$, Ionenquellen-Druck $10^{-6}-2 \times 10^{-6}$ Torr, Analysator-Druck $<10^{-6}$. 
Die Zerfalle metastabiler Molekull-Ionen wurden am gleichen Gerăt mit Hilfe der DADITechnik ${ }^{11)}$ untersucht. Die freigesetzte kinetische Energie $T$ wurde aus der Signalbreite für den entsprechenden Zerfall mit Korrektur fur die Signalbreite des Haupt-Ionenstrahls ${ }^{29}$ ) berechnet. Zur Auswertung wurde die Signalform durch ein Dreieck oder Trapez wiedergegeben und die Basis-Breite bestimmt. Die angegebenen $T_{\mathbf{B}}$-Werte sind Mittelwerte aus mindestens 3 Messungen. Reproduzierbarkeit $< \pm 10 \%$.

lonisierungs- und Auftrittsenergien wurden mit einem für diese Messungen modifizierten Massenspektrometer 6) Vacuum Generators MM 12 B bestimmt (Emissionsstrom $20 \mu$ A, PusherPotential $O$ V). Als Referenzsubstanz wurde Methyliodid $\left(I(\mathrm{M})=9.53 \mathrm{eV}^{23}\right)$ ) gleichzeitig eingelassen. Die Ionenausbeute-Kurven wurden graphisch nach der "semilog-plot"-Methode ${ }^{30}$ ) ausgewertet. Die angegebenen Werte sind Mittelwerte aus mindestens drei Messungen. Reproduzierbarkeit: $I(\mathrm{M}) \pm 0.05 \mathrm{eV} ; A\left(\mathrm{~F}^{+}\right)< \pm 0.1 \mathrm{eV}$.

MI-Spektren und CA-Spektren wurden mit einem Massenspektrometer Vacuum Generators $\mathrm{ZAB}-2 \mathrm{~F}$ unter folgenden Bedingungen erhalten: Elektronenenergie $70 \mathrm{eV}$, Emissionsstrom $200 \mu \mathrm{A}$, Beschleunigungsspannung $8 \mathrm{kV}$, Massenauflosungsvermogen $\approx 3000$, Temperatur der Ionenquelle $180-200^{\circ} \mathrm{C}$, Druck in der Ionenquelle $5 \times 10^{-6}$ Torr. Das Geralt ist mit Stoßzellen im 1. und 2. feldfreien Raum ausgerustet. Zur Aufnahme der CA-Spektren wurde in die betreffende Stoßzelle ein He-Strom eingelassen, bis die Intensitat des Haupt-Ionenstrahls auf ca. 10\% des ursprünglichen Wertes abgesunken war. Reproduzierbarkeit der Intensitätsverhältnisse in den MI-Spektren und den CA-Spektren (mit Ausnahme der Signale für unimolekulare Zerfälle) $< \pm 10 \%$.

\section{Darstellung der Substanzen:}

Die trans-2-Stilbazole [2-(trans-2-Phenylethenyl)pyridine] $1 \mathrm{a}-\mathrm{g}$ und trans-4-Stilbazol [4-(trans2-Phenylethenyl)pyridin] (3) wurden nach Standardvorschriften ${ }^{31)}$ durch Kondensation von 2- bzw. 4-Methylpyridin mit entsprechend substituierten Benzaldehyden in Acetanhydrid (Methode A) oder Umsetzen von N,2-Dimethylpyridinium-iodid mit substituierten Benzaldehyden in Methanol/Piperidin und anschließender Pyrolyse i. Vak. (Methode B) hergestellt. Die Verbindungen wurden dünnschichtchromatographisch und spektroskopisch auf Reinheit gepruft.

cis-2-Stilbazol 31 b), trans-3-Stilbazol (2) ${ }^{32)}$, und 4-Azaphenanthren (6) ${ }^{33)}$ (Benzo[h]chinolin) wurden nach Literaturvorschriften synthetisiert.

Tab. 7. Ausbeuten, Schmelzpunkte und Analysen der untersuchten Verbindungen

\begin{tabular}{|c|c|c|c|c|c|c|c|c|}
\hline & Methode & $\begin{array}{c}\text { Ausb. } \\
(\%)\end{array}$ & $\underset{\text { (Lit.) }}{\text { Schmp. }\left({ }^{\circ} \mathrm{C}\right)}$ & $\begin{array}{l}\text { Summenformel } \\
\text { (Molmasse) }\end{array}$ & & $\mathrm{C}^{A}$ & $\begin{array}{c}\text { Analyse } \\
\mathrm{H}\end{array}$ & $\mathbf{N}$ \\
\hline $1 \mathbf{a}$ & B & 43 & $\begin{array}{c}92-93 \\
(93)\end{array}$ & $\begin{array}{r}\mathrm{C}_{13} \mathrm{H}_{11} \mathrm{~N} \\
(181.2)\end{array}$ & $\begin{array}{l}\text { Ber. } \\
\text { Gef. }\end{array}$ & $\begin{array}{l}86.15 \\
85.67\end{array}$ & $\begin{array}{l}6.12 \\
6.32\end{array}$ & $\begin{array}{l}7.73 \\
7.80\end{array}$ \\
\hline 16 & A & 53 & $71-72$ & $\begin{array}{c}\mathrm{C}_{13} \mathrm{H}_{10} \mathrm{FN} \\
(199.2)\end{array}$ & $\begin{array}{l}\text { Ber. } \\
\text { Gef. }\end{array}$ & $\begin{array}{l}78.46 \\
79.02\end{array}$ & $\begin{array}{l}5.07 \\
5.41\end{array}$ & $\begin{array}{l}7.04 \\
7.16\end{array}$ \\
\hline $1 \mathrm{c}$ & B & 37 & - & $\begin{array}{r}\mathrm{C}_{14} \mathrm{H}_{13} \mathrm{~N} \\
(195.3)\end{array}$ & & & & \\
\hline $1 d$ & A & 55 & $76-77$ & $\begin{array}{c}\mathrm{C}_{13} \mathrm{H}_{10} \mathrm{ClN} \\
(215.2)\end{array}$ & $\begin{array}{l}\text { Ber. } \\
\text { Gef. }\end{array}$ & $\begin{array}{l}72.57 \\
72.06\end{array}$ & $\begin{array}{l}4.68 \\
4.80\end{array}$ & $\begin{array}{l}6.51 \\
6.59\end{array}$ \\
\hline $1 \mathrm{e}$ & A & 60 & $76-77$ & $\begin{array}{c}\mathrm{C}_{13} \mathrm{H}_{10} \mathrm{BrN} \\
(259.6)\end{array}$ & $\begin{array}{l}\text { Ber. } \\
\text { Gef. }\end{array}$ & $\begin{array}{l}60.15 \\
59.79\end{array}$ & $\begin{array}{l}3.88 \\
4.16\end{array}$ & $\begin{array}{l}5.40 \\
5.44\end{array}$ \\
\hline $1 \mathrm{f}$ & $\mathbf{A}$ & 60 & $64-65$ & $\begin{array}{c}\mathrm{C}_{13} \mathrm{H}_{10} \mathrm{IN} \\
(307.1)\end{array}$ & $\begin{array}{l}\text { Ber. } \\
\text { Gef. }\end{array}$ & $\begin{array}{l}50.84 \\
50.43\end{array}$ & $\begin{array}{l}3.28 \\
3.08\end{array}$ & $\begin{array}{l}4.56 \\
4.46\end{array}$ \\
\hline $1 \mathrm{~g}$ & $\mathbf{A}$ & 70 & $100-101$ & $\begin{array}{c}\mathrm{C}_{13} \mathrm{H}_{10} \mathrm{~N}_{2} \mathrm{O}_{2} \\
(226.2)\end{array}$ & $\begin{array}{l}\text { Ber. } \\
\text { Gef. }\end{array}$ & $\begin{array}{l}69.09 \\
69.10\end{array}$ & $\begin{array}{l}4.46 \\
4.61\end{array}$ & $\begin{array}{l}12.40 \\
12.59\end{array}$ \\
\hline
\end{tabular}


Abschätzung von $\Delta H_{B}(\mathbf{a})$ aus thermochemischen Daten: Die Bildung der lonen a aus 1 a kann durch die hypothetische Reaktionsfolge a) - d) (Schema 3) beschrieben werden:

\section{Schema 3}

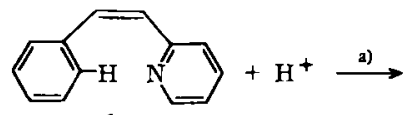

$1 \mathbf{a}$

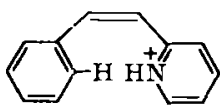

$\mathbf{x}$
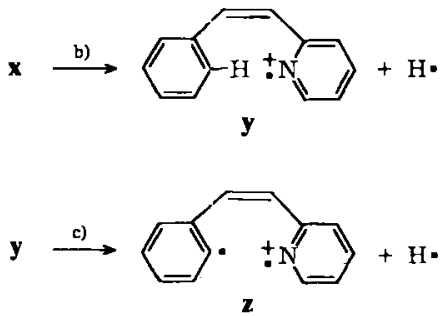

2

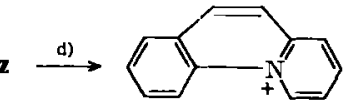

8

Für die einzelnen Reaktionsschritte gilt $(P A=$ Protonen-Affinität):
a) $\mathrm{PA}(1 \mathbf{a})=\Delta H_{\mathrm{B}}(1 \mathbf{a})+\Delta H_{\mathrm{B}}\left(\mathrm{H}^{+}\right)-\Delta H_{\mathrm{B}}(\mathbf{x})$
b) $D(\stackrel{+}{\mathrm{N}}-\mathrm{H})=\Delta H_{\mathrm{B}}(\mathrm{y})+\Delta H_{\mathrm{B}}\left(\mathrm{H}^{-}\right)-\Delta H_{\mathrm{B}}(\mathbf{x})$
c) $D(\mathrm{C}-\mathrm{H})=\Delta H_{\mathrm{B}}(\mathrm{z})+\Delta H_{\mathrm{B}}\left(\mathrm{H}^{*}\right)-\Delta H_{\mathrm{B}}(\mathrm{y})$
d) $D(\stackrel{+}{\mathbf{N}}-\mathrm{Ph})+\Delta E_{\mathrm{Del}}=\Delta H_{\mathrm{B}}(\mathbf{z})-\Delta H_{\mathrm{B}}(\mathrm{a})$

Daraus folgt:

$$
\begin{aligned}
\Delta H_{\mathrm{B}}(\mathrm{a})=\Delta H_{\mathrm{B}}(1 \mathrm{a}) & +\Delta H_{\mathrm{B}}\left(\mathrm{H}^{+}\right)-2 \Delta H_{\mathrm{B}}\left(\mathrm{H}^{-}\right)-\mathrm{PA}(1 \mathrm{a})+D(\mathrm{C}-\mathrm{H}) \\
& +[D(\stackrel{+}{\mathrm{N}}-\mathrm{H})-D(\stackrel{\mathrm{N}}{\mathrm{N}}-\mathrm{Ph})]-\Delta E_{\mathrm{Del}}
\end{aligned}
$$

Die Bildungsenthalpien $\Delta H_{\mathrm{B}}(1 \mathrm{a})=297 \mathrm{~kJ} \cdot \mathrm{mol}^{-1}$ (aus Inkrementen $\left.{ }^{18)}\right), \Delta H_{\mathrm{B}}\left(\mathrm{H}^{+}\right)=1528$ $\mathrm{kJ} \cdot \mathrm{mol}^{-1}{ }^{23)}$ und $\Delta H_{\mathrm{B}}\left(\mathrm{H}^{*}\right)=218 \mathrm{~kJ} \cdot \mathrm{mol}^{-1}$ 23) sind bekannt. $D(\mathrm{C}-\mathrm{H})=436 \mathrm{~kJ} \cdot \mathrm{mol}^{-1}{ }^{34}$ entspricht der Dissoziationsenergie einer $\mathrm{C}-\mathrm{H}$-Bindung in Benzol. Die Differenz der Dissoziationsenergien für die homolytische Spaltung einer $\mathbf{N}-\mathrm{H}$-Bindung in Ammonium-Verbindungen, $D(\stackrel{+}{\mathbf{N}}-\mathrm{H})-D(\stackrel{+}{\mathbf{N}}-\mathrm{Ph})$, ist $\mathrm{zu}+20 \mathrm{kj} \cdot \mathrm{mol}^{-1}$ abgeschätzt worden 7 . Die Protonen-Affinität von $1 \mathrm{a}, \mathrm{PA}(1 \mathrm{a})$, ist nicht bekannt.

In der Gasphase wird die Anlagerung eines Protons an das freie Elektronenpaar des N-Atoms im Pyridin durch die wenig polare Styrylgruppe voraussichtlich wenig beeinflußt, so daß in der 1. Năherung gilt PA(1 a $)=\operatorname{PA}($ Pyridin $)=916 \mathrm{~kJ} \cdot \mathrm{mol}^{-1}\left(219 \mathrm{kcal} \mathrm{mol}^{-1}{ }^{35)}\right) \cdot \Delta E_{\text {Del }}$ ist die Differenz der Delokalisierungsenergien von $\mathrm{a}$ und $\mathrm{z}$ in Gleichung d), die durch die entsprechende Differenz bei Phenanthren und Stilben wiedergegeben werden kann. Nach theoretischen Berechnungen ${ }^{36)}$ betragt sie $26 \mathrm{~kJ} \cdot \mathrm{mol}^{-1}(0.27 \mathrm{eV})$. Nach Einsetzen dieser Werte wird $\Delta H_{\mathrm{B}}(\mathrm{a})$ $=903 \mathrm{~kJ} \cdot \mathrm{mol}^{-1}$ erhalten. 
Dieser Wert ist jedoch mit großer Wahrscheinlichkeit etwas zu hoch, weil wegen des stabilisierenden Effektes von großen, polarisierbaren Substituenten auf Pyridinium-Ionen PA(1a) > PA(Pyridin) sein sollte. Bei substituierten Pyridinen ist sowohl für die Ionisierungsenergie des Elektronenpaars am N-Atom ${ }^{37}$ ) als auch für die Protonen-Affinität ${ }^{38)}$ eine Abhängigkeit von den $\sigma^{+}$-Konstanten beobachtet worden. Daraus läßt sich PA(1 a) - PA(Pyridin) $\approx 8 \mathrm{~kJ} \cdot \mathrm{mol}^{-1}$ abschätzen, so daß sich ein wahrscheinlicher Wert $\Delta H_{\mathrm{B}}(\mathrm{a})=895 \mathrm{~kJ} \cdot \mathrm{mol}^{-1}$ ergibt.

\section{Literatur}

1) Mechanismen massenspektrometrischer Fragmentierungsreaktionen, XXVII; Teil XXVI: $R$. Schubert und H. F. Grützmacher, J. Am. Chem. Soc. 102, 5323 (1980).

2) D. H. Williams, Acc. Chem. Res. 10, 280 (1977).

3) R. G. Cooks, J. H. Beynon, R. M. Caprioli und G. R. Lester, Metastable Ions, Elsevier, Amsterdam 1973.

4) R. G. Cooks, M. Bertrand, J. H. Beynon, M. E. Rennekamp und D. W. Setser, J. Am. Chem. Soc. 95, 1732 (1973).

5) Sa) J. R. Christie, P. J. Derrick und G. J. Rickard, J. Chem. Soc., Faraday Trans. 2, 304 (1978). - ${ }^{\text {b) }}$ D. A. Krause, R. J. Day, W. L. Jorgensen und R. G. Cooks, Int. J. Mass Spectrom. Ion Phys. 27, 227 (1978). - ${ }^{\text {Sc) }}$ R. J. Day, D. A. Krause, W. L. Jorgensen und $R . G$. Cooks, ebenda 30, 83 (1979).

6) B. Schaldach und H. F. Grützmacher, Int. J. Mass Spectrom. Ion Phys. 31, 271 (1979).

7) $R$. Schubert und H. F. Grützmacher, Org. Mass Spectrom. 15, 122 (1979).

8) R. A. W. Johnstone und S. D. Ward, J. Chem. Soc. C 1968, 2540.

9) T. W. Shannon und F. W. McLafferty, J. Am. Chem. Soc. 88, 5021 (1966).

10) A. P. Bruins, K. R. Jennings und S. Evans, Int. J. Mass Spectrom. Ion Phys. 26, 395 (1978).

11) 11a) J. H. Beynon, R. M. Caprioli und T. Ast, Org. Mass Spectrom. 5, 229 (1971). - 11b) K. H. Maurer, C. Brunnée, G. Kappus, K. Habfast, U. Schröder und P. Schulze; XIX. Ann. Conf. Mass Spectrom. Allied Topics, Atlanta, Ga. 1971.

12) B. Schaldach und H. F. Grützmacher, Org. Mass Spectrom. 15, 166 (1980).

13) Wegen der kürzeren Reaktionszeit besitzen im 1. feldfreien Raum zerfallende Ionen eine höhere Anregungsenergie als solche, deren Zerfälle im 2. feldfreien Raum beobachtet werden. Dies erklärt die Unterschiede der in beiden feldfreien Räumen gemessenen MI-Spektren (Tab. 2), bei denen die energetisch günstigere Abspaltung von $\mathrm{HCN}$ zu Produkt-Ionen $m / z=153$ bei den Reaktionen der energiearmen Ionen im 2. feldfreien Raum stärker hervortritt.

14) 14a) W. F. Haddon und F. W. McLafferty, J. Am. Chem. Soc. 90, 4745 (1968). - ${ }^{14 b)} K . R$. Jennings, Int. J. Mass Spectrom. Ion Phys. 1, 227 (1968).

15) CA-Spektren entstehen ebenso wie „normale“ Massenspektren durch schnelle Zerfälle hochangeregter lonen. Ebenso wie bei den Massenspektren stellungsisomerer aromatischer Verbindungen sind daher auch für die CA-Spektren der isomeren $\mathrm{C}_{13} \mathrm{H}_{10} \mathrm{~N}^{+}$-Ionen nur relativ geringe quantitative Unterschiede zu erwarten.

16) J. F. Elder jr., J. H. Beynon und R. G. Cooks, Org. Mass Spectrom. 11, 415 (1976).

17) T. W. Bentley und R. A. W. Johnstone, J. Chem. Soc. B 1971, 263.

18) Die Bildungsenthalpien von $1 \mathrm{a}-\mathrm{g}$ wurden nach der Inkrement-Methode von $S$. W. Benson, $F$. R. Cruickshank, D. M. Golden, G. R. Haugen, H. E. O'Neal, A. S. Rodgers, R. Shaw und R. Walsh, Chem. Rev. 69, 279 (1969) berechnet. Bildungsenthalpien der Radikale $X_{\mathrm{j}}$ siehe Lit. ${ }^{23)}$.

19) $D(\dot{\mathrm{C}}-\mathrm{X})$ entspricht der Dissoziationsreaktion

$\mathrm{XC}_{6} \mathrm{H}_{4}-\mathrm{CH}=\mathrm{CH}-\mathrm{C}_{5} \mathrm{H}_{3} \mathrm{~N}^{\top+} \rightarrow{ }^{+} \mathrm{C}_{6} \mathrm{H}_{4}-\mathrm{CH}=\mathrm{CH}-\mathrm{C}_{5} \mathrm{H}_{3} \mathrm{~N}+\mathrm{X}$.

$\triangle H_{B}\left({ }^{+} \mathrm{C}_{6} \mathrm{H}_{4}-\mathrm{CH}=\mathrm{CH}-\mathrm{C}_{5} \mathrm{H}_{3} \mathrm{~N}\right)$ eines Ions mit positiver Ladung an einem C-Atom der Phenylgruppe ist nicht bekannt, so daß die relativen Dissoziationsenergien $\Delta D(\stackrel{+}{\mathrm{C}}-\mathrm{X})$ in Bezug auf $1 \mathrm{a}(\mathrm{X}=\mathrm{H})$ berechnet wurden nach

$\Delta D(\dot{\mathrm{C}}-\mathrm{X})=\Delta H_{\mathrm{B}}\left(\mathrm{X}_{\mathrm{i}}\right)-\Delta H_{\mathrm{B}}(\mathrm{H})+\Delta H_{\mathrm{B}}\left(1 \mathrm{a}^{+}\right)-\Delta H_{\mathrm{B}}\left(1 \mathrm{i}^{+}\right)$.

20) Zur Ableitung dieser Beziehung siehe Lit. ${ }^{6}$.

21) Beim Zerfall metastabiler Ionen beobachtet man in der Regel für einen bestimmten Prozeß eine charakteristische Verteilung der freigesetzten kinetischen Energie ${ }^{3)}$. Für thermochemische Betrachtungen ist der maximale Wert der freigesetzten kinetischen Energie, $T_{B}$, wichtig, der aus der Basisbreite der MIKE-Signale bestimmt wurde (s. Exp. Teil).

22) s. Lit. ${ }^{16)}$.

23) H. M. Rosenstock, K. Draxl, B. W. Steiner und J. T. Herron, Energetics of Gaseous Ions, J. Phys. Chem. Ref. Data 6, Suppl. 1 (1977). 
24) P. J. Robinson und K. A. Holbrock, Unimolecular Reactions, Wiley-Interscience, New York 1971.

25) H. Kuschel und H. F. Grützmacher, Org. Mass Spectrom. 9, 408 (1974).

26) G. S. Hammond, J. Am. Chem. Soc. 77, 334 (1955).

27) A. R. Miller, J. Am. Chem. Soc. 100, 1984 (1979).

28) J. C. Polanyi, Acc. Chem. Res. 5, 161 (1972).

29) M. A. Baldwin, J. P. Derrick und R. P. Morgan, Org. Mass Spectrom. 11, 440 (1976).

30) F. P. Lossing, A. N. Tickner und W. A. Bryce, J. Chem. Phys. 19, 1254 (1951).

31) 31a) J. L. R. Williams und S. K. Webster, J. Org. Chem, 26, 4893 (1961). - 31b) J. L. R. Williams, R. E. Adel, J. M. Carlson, G. A. Reynolds, D. G. Borden und J. A. Ford, ebenda 28 , 387 (1963).

32) L. Horner, H. Hoffmann, H. G. Wippel und G. Klahre, Chem. Ber. 92, 2499 (1959).

33) C. E. Loader und C. J. Timmons, J. Chem. Soc. C 1966, 1078.

34) J. A. Kerr, Chem. Rev, 66, 465 (1966).

35) B. S. Freiser und J. L. Beauchamp, J. Am. Chem. Soc. 98, 265 (1976).

36) W. C. Herndorn und M. L. Ellzey jr., J. Am. Chem. Soc. 96, 6631 (1974).

37) B. G. Ramsey und F. A. Walker, J. Am. Chem. Soc. 96, 3314 (1974).

38) N. Taagepera, W. G. Henderson, R. T. C. Brownlee, J. L. Beauchamp, D. Holtz und R.W. Taft, J. Am. Chem. Soc. 94, 1369 (1972). 